\title{
A Cooperative Game of Information Trading: The Core, the Nucleolus and the Kernel
}

\author{
By T. Driessen ${ }^{1}$, S. Muto ${ }^{2}$, and M. Nakayama ${ }^{3}$
}

\begin{abstract}
A certain trade of the information about a technological innovation between the initial owner of the information and $n$ identical producers is studied by means of a cooperative game theoretic approach. The information trading situation is modelled as a cooperative $(n+1)$-person game with side payments. The symmetrical strong $\varepsilon$-cores (including the core), the nucleolus and the kernel of the cooperative game model are determined. Interpretations of these game theoretic solutions and their implications for the information trading problem are given.
\end{abstract}

\section{An Economic Model of Information Trading}

We consider an industry consisting of a fixed number of identical producers of which all produce the same homogeneous output with the same technology. The profit level of each producer is identical and expressed in terms of monetary units. In addition to the producers there is another agent who possesses the information about a technological innovation. The new technology may increase the monetary profit level of the producers who acquire and utilize it, but may decrease the profit level of the producers who don't purchase it and continue to use the old technology. Due to an external diseconomy, the profit level of every producer decreases as the number of producers who purchase the new technology grows. The initial owner of the information about the technological innovation has no means of production. As such, we regard the initial owner of the information as the seller of the technological innovation and the producers as the potential buyers of the new technology. To be exact, the initial owner attempts to sell the information about the new technology to all or some of the producers. We aim to describe the profit that can be realized by the initial owner of the information if a trade of the information has been carried out. Concerning the pro-

1 Theo Driessen, Department of Applied Mathematics, University of Twente, Enschede, The Netherlands.

2 Shigeo Muto, Faculty of Economics, Tohoku University, Kawauchi, Sendai, Japan.

3 Mikio Nakayama, Department of Economics, Hosei University, Tokyo, Japan. 
ducers, the relevant comparison is between the profit level with the use of the new technology and the profit level obtained before the technological innovation.

Our analysis of the above information trading situation will be based on a cooperative game model in which the players consist of the seller and the potential buyers of the new technology. In other words, we define a so-called cooperative game in characteristic function form in such a way that the corresponding characteristic function reflects the cooperative behaviour between the seller and the potential buyers. In the context of the relevant game theoretic approach, it is permitted that the players communicate with one another in order to make binding agreements regarding the coalition formation. For instance, the producers can make a binding agreement not to purchase the new technology if they want to do so. Further, a trade of the information can only be carried out on the understanding that resales of the new technology are completely prohibited. The exact cooperative game model of the information trading situation will be treated in Section 2.

Following the cooperative setting of Section 2, the profit shares to the seller and the producers can be prescribed by basic solution concepts. The general notions of three main solution concepts - the strong $\varepsilon$-cores (including the core), the nucleolus and the kernel - are discussed in Section 3. Our attention is mainly directed to the core and the nucleolus solution concepts. In the Sections 4, 5 and 6 respectively, we determine the strong $\varepsilon$-cores, the nucleolus (as well as the core) and the kernel of the cooperative game model of the information trading situation. Section 7 deals with the monopolistic structure of the information trading situation and the implications for the solution concepts of the corresponding cooperative game model. Concluding remarks are given in Section 8. The most important results can be summarized as follows.

The symmetrical strong $\varepsilon$-core elements can be described in terms of two realvalued functions $f$ and $g$ of the real number $\varepsilon$ (cf. Lemma 4.2 and Theorem 4.4). The sign of the critical number $\varepsilon^{*}$ satisfying $f\left(\varepsilon^{*}\right)=g\left(\varepsilon^{*}\right)$ determines whether the core is empty or not (cf. Theorem 5.2). According to the nucleolus concept, the profit share to any producer equals either the amount $f\left(\varepsilon^{*}\right)$ or the bound $E^{*}(n-1)$ induced by the individual rationality principle (cf. Theorem 5.1). As a consequence, the core is nonempty if and only if the potential nucleolus profit share $f\left(\varepsilon^{*}\right)$ to any producer exceeds his monetary profit level obtained before the technological innovation. Further, the nonemptiness of the core implies that the new technology is actually acquired by at least one producer or even all producers (cf. Theorem 5.3). Finally, the kernel coincides with the nucleolus (cf. Theorem 6.2) and the monopolistic structure of the information trading situation yields an empty core (cf. Theorem 7.2). 


\section{A Cooperative Game Model of Information Trading}

First of all, we specify four assumptions on the underlying economic model of the information trading situation as presented in Section 1. Recall that the initial owner seeks to sell the information about the technological innovation to all or some of the $n$ producers $(n \geq 2)$.

(A.1) Given any nontrivial number of actual buyers of the new technology, an actual buyer attains at least as many profits as a nonbuyer.

(A.2 - 3) The external diseconomy applies to both the actual buyers and the nonbuyers. That is, the profit level of any type of a producer decreases as the number of actual buyers grows.

(A.4) The profit level of a unique buyer is more than the positive profit level obtained before the technological innovation.

If $t$ producers have purchased the new technology, then $E(t)$ and $E^{*}(t)$ respectively denote the monetary profit level of any producer who has purchased the new technology and not. The collection $\left\{E(t), E^{*}(t) \mid t \in\{0,1, \ldots, n\}\right\}$ is called a profit structure of the information trading situation if it satisfies the above four assumptions, i.e.,
(A.1) $E(t) \geq E^{*}(t)$
for all $t \in\{1,2, \ldots, n-1\}$,
(A.2) $\quad E(t) \geq E(t+1)$
for all $t \in\{1,2, \ldots, n-1\}$,
(A.3) $E^{*}(t) \geq E^{*}(t+1)$ for all $t \in\{0,1, \ldots, n-1\}$,
(A.4) $E(1)>E^{*}(0)>0, E(0)=E^{*}(n):=0$.

For the sake of mathematical convenience, we put $E(0)=E^{*}(n):=0$. Next we model the information trading situation as a cooperative $(n+1)$-person game where its player set $N^{0}$ consists of the seller 0 and the potential buyers $1,2, \ldots, n$ of the new technology. That is, $N^{0}=\{0\} \cup N$ where $N:=\{1,2, \ldots, n\}$ represents the set of all $n$ producers. Any nonempty subset $S$ of the player set $N^{0}$ (notation: $S \subset N^{0}$ ) is called a coalition. A coalition which includes the seller is always denoted by $S^{0}$ in such a way that $S^{0}=\{0\} \cup S$ with $S \subset N$. The number of potential buyers in a coalition $S^{0}$ and $S$ is denoted by $S$.

The cornerstone of the cooperative game model is the so-called characteristic function $v: 2^{N^{0}} \rightarrow \mathbb{R}$ which assigns to every coalition its maximal joint profit. To be exact, the worth $v\left(S^{0}\right)$ of any coalition $S^{0}$ represents the largest possible monetary profit what the producers in $S^{0}$ can achieve by the cooperative behaviour between themselves and the initial owner of the information about the technological innovation. Notice that the coalition $S^{0}$ must determine the profit independently of the members of the complementary coalition $N^{0}-S^{0}$ because 
resales of the new technology are completely prohibited. In point of fact, the new technology is acquired and utilized by a suitable number of producers in $S^{0}$ so as to maximize the joint profit of production. Moreover, if the initial owner of the information is not a member of a coalition $S$, then the producers in $S$ can not acquire the new technology by cooperation within $S$. Consequently, the worth $v(S)$ of any coalition $S$ represents the joint profit of production under the worst conceivable circumstance that all producers outside $S$ do purchase the new technology.

Definition 2.1: The cooperative game $\left(N^{0} ; v\right)$ of the information trading situation is given by

$$
\begin{aligned}
& v\left(S^{0}\right):=\max \left(t E(t)+(s-t) E^{*}(t) \mid t \in\{0,1, \ldots, s\}\right) \text { for all } S^{0} \subset N^{0}, \\
& v(S):=s E^{*}(n-s) \text { for all } S \subset N .
\end{aligned}
$$

Obviously, the above information trading game $\left(N^{0} ; v\right)$ satisfies

$$
\begin{aligned}
& v\left(N^{0}\right) \geq n E^{*}(0)=v(N), \quad v(\{0\})=0, \\
& v(\{i\})=E^{*}(n-1), \quad v(\{0, i\})=E(1) \quad \text { for all } \quad i \in N .
\end{aligned}
$$

\section{Game Theoretic Notions}

The profit shares to the seller and the $n$ producers will be described by means of a specific $(n+1)$-dimensional payoff vector $x=\left(x_{0}, x_{1}, \ldots, x_{n}\right) \in \mathbb{R}^{n+1}$ where $x_{i}$ represents the payoff (the profit share) to player $i$. Following the cooperative game model of Section 2 and the solution part of cooperative game theory, it is customary to require that any payoff vector meets the efficiency and individual rationality principles. In other words, any payoff vector for the information trading game $\left(N^{0} ; v\right)$ should belong to the imputation set $I(v)$ which is defined to be

$$
\begin{aligned}
I(v):=\left\{\left(x_{0}, x_{1}, \ldots, x_{n}\right) \in \mathbb{R}^{n+1} \mid \sum_{j \in N^{0}} x_{j}=v\left(N^{0}\right)\right. \text { and } \\
\left.\left.x_{i} \geq v(i i\}\right) \text { for all } i \in N^{0}\right\}
\end{aligned}
$$


or equivalently,

$$
\begin{array}{r}
I(v):=\left\{\left(x_{0}, x_{1}, \ldots, x_{n}\right) \in \mathbb{R}^{n+1} \mid \sum_{j \in N^{0}} x_{j}=v\left(N^{0}\right), \quad x_{0} \geq 0\right. \text { and } \\
\left.x_{i} \geq E^{*}(n-1) \text { for all } i \in N\right\} .
\end{array}
$$

Note that the imputation set $I(v)$ of the information trading game is nonempty since $v\left(N^{0}\right) \geq(n-1) E(n-1)+E^{*}(n-1) \geq n E^{*}(n-1)$ by using Definition 2.1 and assumption A.1. The prescription of one or more specific imputations by basic solution concepts is mainly based on the notion of excess. The excess of the coalition $T$ with respect to the payoff vector $x$ in the information trading game $\left(N^{0} ; v\right)$ is defined to be

$$
e(T, x):=v(T)-\sum_{j \in T} x_{j}=v(T)-x(T)
$$

For the sake of notational convenience, we always write $x(T)$ instead of $\sum_{j \in T} x_{j}$. A nonnegative (nonpositive respectively) excess of $T$ at $x$ represents the gain (loss) to the coalition $T$ if its members withdraw from the payoff vector $x$ in order to form their own coalition.

Our main goal is to determine and discuss the profit shares to the seller and the producers on the basis of the "nucleolus" solution concept. The nucleolus of any cooperative game is a specific imputation of which the formal definition is given in terms of excess and "minimum complaint". For any imputation $x \in \mathbb{R}^{n+1}$ in the information trading game $\left(N^{0} ; v\right)$, let the associated complaint vector $\theta(x)$ be defined as the $2^{n+1}$-tuple whose components are the excesses $e(T, x), T \subset N^{0}$, arranged in nonincreasing order. The nucleolus of the information trading game $\left(N^{0} ; v\right)$ consists of imputations that minimize the complaint function $\theta(\cdot)$ of $x$ in the lexicographic order on $\mathbb{R}^{2^{n+1}}$ over the nonempty compact convex imputation set $I(v)$. It is well-known that the nucleolus of any cooperative game is always a singleton (cf. Schmeidler, 1969). The unique point in the nucleolus of the information trading game $\left(N^{0} ; v\right)$ is denoted by $\eta(v)$.

Generally speaking, the above formal definition of the nucleolus is useless for its determination. Our approach to the exact determination of the nucleolus of the information trading game will be guided by the position of the nucleolus within any nonempty "strong $\varepsilon$-core". Informally, the strong $\varepsilon$-core of any cooperative game consists of efficient payoff vectors that give rise only to ex- 
cesses not greater than $\varepsilon$ for all coalitions different from the grand coalition. Formally, the notion of a strong $\varepsilon$-core is described as follows (cf. Shapley and Shubik, 1963, 1966).

Definition 3.1: For any $\varepsilon \in \mathbb{R}$, the strong $\varepsilon$-core $C_{\varepsilon}(v)$ of the information trading game $\left(N^{0} ; v\right)$ is given by

$$
\begin{aligned}
C_{\varepsilon}(v):=\left\{x \in \mathbb{R}^{n+1} \mid\right. & x\left(N^{0}\right)=v\left(N^{0}\right) \text { and } \\
& \left.x(T) \geq v(T)-\varepsilon \text { for all } T \subset N^{0}, T \neq N^{0}, \phi\right\} .
\end{aligned}
$$

Evidently, the strong $\varepsilon$-core is nonempty (empty respectively) if the real number $\varepsilon$ is large (small) enough. As a matter of fact, the inclusion $C_{\delta}(v) \subset C_{\varepsilon}(v)$ holds whenever $\delta<\varepsilon$, with strict inclusion if $C_{\varepsilon}(v) \neq \phi$. Due to its formal definition, the nucleolus of any cooperative game is always contained in the nonempty intersection of any strong $\varepsilon$-core with the imputation set (cf. Schmeidler, 1969).

Theorem 3.2: Let $\left(N^{0} ; v\right)$ be the information trading game. Then

$$
\eta(v) \in C_{\varepsilon}(v) \cap I(v) \text { whenever } \quad C_{\varepsilon}(v) \cap I(v) \neq \phi .
$$

In Section 4 we describe both the structure of any strong $\varepsilon$-core and the intersection of any strong $\varepsilon$-core with the imputation set. The above theorem is of the highest importance for the exact determination of the nucleolus of the information trading game (see Section 5). In Section 6 we determine the "kernel", a solution concept which is closely related to the nucleolus. Davis and Maschler (1965) introduced the kernel of any cooperative game in terms of excess and "maximum surplus". In this paper we omit the formal definition of the kernel because we prefer to exploit the geometric characterization for the kernel as presented in Maschler, Peleg and Shapley (1979). In point of fact, we are interested in the geometric structure of the intersections of the kernel with the strong $\varepsilon$-cores. The main idea in the relevant geometric approach is as follows.

Consider a fixed strong $\varepsilon$-core element $x$ and two arbitrary players $i, j$. Let the $i$-th coordinate of $x$ decrease and the $j$-th coordinate of $x$ increase by the same nonnegative amount. Let the nonnegative real number $\delta_{i j}(\varepsilon, x)$ represent the largest amount that can be transferred from player $i$ to player $j$ with respect to the strong $\varepsilon$-core element $x$ while remaining in the strong $\varepsilon$-core. The critical number $\delta_{i j}(\varepsilon, x)$ is well-defined because any nonempty strong $\varepsilon$-core is a compact convex set. The next theorem treats the bisection property for the intersection of the kernel $K(v)$ with a nonempty strong $\varepsilon$-core $C_{\varepsilon}(v)$ of the information trading game $\left(N^{0} ; v\right)$. 
Definition 3.3: Let $\varepsilon \in \mathbb{R}, x \in C_{\varepsilon}(v)$ and $i, j \in N^{0}, i \neq j$.

Let $e^{i} \in \mathbb{R}^{n+1}, e^{j} \in \mathbb{R}^{n+1}$ denote the $i$-th and $j$-th unit vector. The critical number $\delta_{i j}(\varepsilon, x) \in \mathbb{R}$ is given by

$$
\delta_{i j}(\varepsilon, x):=\max \left(\delta \mid \delta \in \mathbb{R}, x-\delta e^{i}+\delta e^{j} \in C_{\varepsilon}(v)\right) .
$$

Theorem 3.4: Let $\varepsilon \in \mathbb{R}$ and $x \in C_{\varepsilon}(v)$. Then

$$
\begin{array}{ll}
x \in K(v) \cap C_{\varepsilon}(v) \text { iff } \quad x \in I(v) \text { and for all } i, j \in N^{0}, i \neq j, \\
\text { either } \quad \delta_{i j}(\varepsilon, x)=\delta_{j i}(\varepsilon, x) \\
\text { or } \left.\quad \delta_{i j}(\varepsilon, x)>\delta_{j i}(\varepsilon, x) \text { and } x_{i}=v((i\})\right) \\
\text { or } \left.\quad \delta_{i j}(\varepsilon, x)<\delta_{j i}(\varepsilon, x) \text { and } x_{j}=v(j\}\right) .
\end{array}
$$

We say an imputation $x \in I(v)$ is interior to the imputation set $I(v)$ whenever $x_{i}>v((i))$ for all $i \in N^{0}$. The bisection property for the intersection of the kernel with a nonempty strong $\varepsilon$-core states that a strong $\varepsilon$-core element $x$ which is interior to the imputation set, belongs to the kernel if and only if the element $x$ is always the midpoint of the largest straight line segments through $x$ in the various directions that are still included in the strong $\varepsilon$-core. It is well-known that the kernel of any cooperative game is a (multi-) point set which always contains the nucleolus (cf. Schmeidler, 1969).

\section{The Strong $\varepsilon$-Cores of the Information Trading Game}

Recall that the $n$ producers are supposed to be identical. We say the producers are symmetrical players in the information trading game $\left(N^{0} ; v\right)$. For that reason, we are merely interested in strong $\varepsilon$-core elements $x \in \mathbb{R}^{n+1}$ that satisfy the additional symmetry requirement $x_{i}=x_{j}$ for all $i, j \in N$. Thus we only consider symmetrical efficient payoff vectors which are defined by $x(\alpha):=\left(v\left(N^{0}\right)-\right.$ $n \alpha, \alpha, \ldots, \alpha) \in \mathbb{R}^{n+1}$ where $\alpha \in \mathbb{R}$. In order to describe a lower and upper bound for the producer's payoff according to symmetrical strong $\varepsilon$-core elements, we first introduce the following two real-valued functions of $\varepsilon$.

Definition 4.1: Let $\left(N^{0} ; v\right)$ be the information trading game. The functions $f$, $g: \mathbb{R} \rightarrow \mathbb{R}$ are given by 


$$
\begin{aligned}
& f(\varepsilon):=\max \left(E^{*}(n-t)-t^{-1} \varepsilon \mid t \in\{1,2, \ldots, n\}\right), \\
& g(\varepsilon):=\min \left((n-s)^{-1}\left[v\left(N^{0}\right)-v\left(S^{0}\right)+\varepsilon\right] \mid S^{0} \varsubsetneqq N^{0}\right) .
\end{aligned}
$$

An interpretation of the functions $f$ and $g$ is as follows. Concerning any $\varepsilon \in \mathbb{R}$, the idea is to impose either a cost of $\varepsilon$ (if $\varepsilon$ is nonnegative) or a bonus of $-\varepsilon$ (if $\varepsilon$ is nonpositive) on the formation of any coalition $T \neq N^{0}$ in the information trading game $\left(N^{0} ; v\right)$. The producers are rewarded on the basis of the equal division principle. The function $f$ describes the maximal reward to a producer for his cooperative behaviour with respect to the formation of coalitions consisting of producers only. The producer's reward is derived from the earnings of the coalitions to be formed. The function $g$ describes the minimal reward to a producer for his noncooperative behaviour with respect to the formation of coalitions containing the initial owner of the information about the technological innovation. The producer's reward is derived from the remaining parts of the total amount $v\left(N^{0}\right)$ after the formation of the coalitions involved. The first lemma states that the producer's payoff according to symmetrical strong $\varepsilon$-core elements is at least $f(\varepsilon)$ and at most $g(\varepsilon)$.

Lemma 4.2: Let $\varepsilon \in \mathbb{R}$. Then

$$
x(\alpha) \in C_{\varepsilon}(v) \text { iff } f(\varepsilon) \leq \alpha \leq g(\varepsilon) .
$$

Proof: By the three Definitions 3.1, 2.1 and 4.1, we obtain the following equivalences:

$$
\begin{aligned}
& x(\alpha) \in C_{\varepsilon}(v) \\
& \Leftrightarrow(x(\alpha))(T) \geq v(T)-\varepsilon \quad \text { for all } T \subset N^{0}, T \neq N^{0}, \phi \\
& \Leftrightarrow v\left(N^{0}\right)+(s-n) \alpha \geq v\left(S^{0}\right)-\varepsilon \quad \text { for all } \quad S^{0} \varsubsetneqq N^{0} \text {, } \\
& \text { and } s \alpha \geq s E^{*}(n-s)-\varepsilon \quad \text { for all } S \subset N, S \neq \phi \\
& \Leftrightarrow \alpha \leq(n-s)^{-1}\left[v\left(N^{0}\right)-v\left(S^{0}\right)+\varepsilon\right] \text { for all } S^{0} \varsubsetneqq N^{0} \text {, } \\
& \text { and } \alpha \geq E^{*}(n-t)-t^{-1} \varepsilon \quad \text { for all } t \in\{1,2, \ldots, n\} \\
& \Leftrightarrow f(\varepsilon) \leq \alpha \leq g(\varepsilon) \text {. }
\end{aligned}
$$


Our purpose is to describe the smallest nonempty strong $\varepsilon$-core that intersects the imputation set at a unique symmetrical imputation. The main results are treated in the next theorem and are proved with the aid of the properties of the functions $f$ and $g$ as listed in the second lemma.

\section{Lemma 4.3:}

(i) $f, g: \mathbb{R} \rightarrow \mathbb{R}$ are continuous functions;

$f$ is strictly decreasing, i.e., $\delta<\varepsilon$ implies $f(\delta)>f(\varepsilon)$;

$g$ is strictly increasing, i.e., $\delta<\varepsilon$ implies $g(\delta)<g(\varepsilon)$.

(ii) $f(\varepsilon)=E^{*}(0)-n^{-1} \varepsilon$ for all $\varepsilon \geq 0$.

(iii) There exists a unique $\varepsilon^{*} \in \mathbb{R}$ with $f\left(\varepsilon^{*}\right)=g\left(\varepsilon^{*}\right)$.

(iv) If $\varepsilon^{*}>n\left[E^{*}(0)-E^{*}(n-1)\right]$, then there exists a unique $\bar{\varepsilon} \in\left(\varepsilon^{*}, \infty\right)$ with $g(\bar{\varepsilon})=E^{*}(n-1)$.

Proof: (i) Clearly, $f$ ( $g$ respectively) is a continuous and strictly decreasing (increasing) function of $\varepsilon$ because it is defined as maximum (minimum) of a finite number of continuous and strictly decreasing (increasing) functions of $\varepsilon$.

(ii) Let $\varepsilon \geq 0$ and $t \in\{1,2, \ldots, n\}$. Then $E^{*}(0) \geq E^{*}(n-t)$ by Assumption A.3 and so, $E^{*}(n-t)-t^{-1} \varepsilon \leq E^{*}(0)-t^{-1} \varepsilon \leq E^{*}(0)-n^{-1} \varepsilon$. It follows that $f(\varepsilon)=$ $E^{*}(0)-n^{-1} \varepsilon$ for all $\varepsilon \geq 0$.

(iii) Define the function $h: \mathbb{R} \rightarrow \mathbb{R}$ by $h(\varepsilon):=f(\varepsilon)-g(\varepsilon)$ for all $\varepsilon \in \mathbb{R}$. From part (i) we deduce that $h$ is a continuous and strictly decreasing function of $\varepsilon$. Further, it is obvious that the function $h$ satisfies both $h(\varepsilon) \uparrow \infty$ if $\varepsilon \downarrow-\infty$ and $h(\varepsilon) \downarrow-\infty$ if $\varepsilon \uparrow \infty$. By applying the intermediate value theorem to the function $h$, we conclude that there exists a unique $\varepsilon^{*} \in \mathbb{R}$ with $h\left(\varepsilon^{*}\right)=0$. This proves the statement (iii).

(iv) Suppose $\varepsilon^{*}>n\left[E^{*}(0)-E^{*}(n-1)\right]$. Then $\varepsilon^{*}>0$ and so, the parts (ii) (iii) yield $g\left(\varepsilon^{*}\right)=f\left(\varepsilon^{*}\right)=E^{*}(0)-n^{-1} \varepsilon^{*}$. We obtain that $g$ is a continuous and strictly increasing function of $\varepsilon$ with $g\left(\varepsilon^{*}\right)<E^{*}(n-1)$ and $g(\varepsilon) \uparrow \infty$ if $\varepsilon \uparrow \infty$. By applying the intermediate value theorem to the function $g$, we conclude that there exists a unique $\bar{\varepsilon} \in\left(\varepsilon^{*}, \infty\right)$ with $g(\bar{\varepsilon})=E^{*}(n-1)$.

\section{Theorem 4.4:}

(i) $x(\alpha) \in C_{\varepsilon^{*}}(v)$ iff $\alpha=f\left(\varepsilon^{*}\right)$.

(ii) If $\varepsilon^{*} \leq n\left[E^{*}(0)-E^{*}(n-1)\right]$, then $x(\alpha) \in C_{E^{*}}(v)$ implies $x(\alpha) \in I(v)$.

(iii) If $\varepsilon^{*}>n\left[E^{*}(0)-E^{*}(n-1)\right]$, then $x(\alpha) \in C_{\bar{\varepsilon}}(v) \cap I(v)$ iff $\alpha=E^{*}(n-1)$.

Proof: The statement (i) follows immediately from Lemma 4.2 and $f\left(\varepsilon^{*}\right)=$ $g\left(\varepsilon^{*}\right)$. Concerning the remaining two statements, we already remark that the equivalence $x(\alpha) \in I(v) \Leftrightarrow E^{*}(n-1) \leq \alpha \leq n^{-1} v\left(N^{0}\right)$ holds. 
(ii) Suppose $\varepsilon^{*} \leq n\left[E^{*}(0)-E^{*}(n-1)\right]$. In case $\varepsilon^{*} \leq 0$, then the inclusions $C_{\varepsilon^{*}}(v) \subset C_{0}(v) \subset I(v)$ hold. So, we may also suppose $\varepsilon^{*}>0$. Now we obtain $f\left(\varepsilon^{*}\right)=E^{*}(0)-n^{-1} \varepsilon^{*} \geq E^{*}(n-1)$ and $f\left(\varepsilon^{*}\right)=E^{*}(0)-n^{-1} \varepsilon^{*}<E^{*}(0) \leq$ $n^{-1} v\left(N^{0}\right)$. In other words, $x(\alpha) \in I(v)$ for $\alpha=f\left(\varepsilon^{*}\right)$. In view of part (i), this proves the statement (ii).

(iii) Suppose $\varepsilon^{*}>n\left[E^{*}(0)-E^{*}(n-1)\right]$. Then $\varepsilon^{*}>0$ and by Lemma 4.3 (iv), we have $\bar{\varepsilon}>\varepsilon^{*}$ as well as $g(\bar{\varepsilon})=E^{*}(n-1)$. From $\bar{\varepsilon}>\varepsilon^{*}>0$ we deduce $f(\bar{\varepsilon})=$ $E^{*}(0)-n^{-1} \bar{\varepsilon}<E^{*}(0)-n^{-1} \varepsilon^{*}<E^{*}(n-1)$. Due to Lemma 4.2, the equivalence $x(\alpha) \in C_{\bar{\varepsilon}}(v) \Leftrightarrow f(\bar{\varepsilon}) \leq \alpha \leq g(\bar{\varepsilon})$ holds, whereas the inequality $E^{*}(n-1) \leq$ $n^{-1} v\left(N^{\gamma}\right)$ always holds. Now we conclude that $x(\alpha) \in C_{\bar{\varepsilon}}(v) \cap I(v)$ if and only if $\alpha=g(\bar{\varepsilon})=E^{*}(n-1)$.

\section{The Nucleolus and the Core of the Information Trading Game}

A well-known property for the nucleolus concept states that symmetrical players in any cooperative game receive the same payoff according to the nucleolus of the game. Particularly, the symmetrical producers in the information trading game $\left(N^{0} ; v\right)$ get the same nucleolus payoff, i.e., $\eta_{i}(v)=\eta_{j}(v)$ for all $i, j \in N$. In view of the crucial Theorem 3.2, the nucleolus of the information trading game will be determined as the unique symmetrical imputation that is still contained in the nonempty intersection of a suitably chosen strong $\varepsilon$-core with the imputation set. For that purpose, we must distinguish two cases as indicated in Theorem 4.4.

Theorem 5.1: Let $\left(N^{0} ; v\right)$ be the information trading game.

(i) If $\varepsilon^{*} \leq n\left[E^{*}(0)-E^{*}(n-1)\right]$, then the nucleolus $\eta(v)=\left(v\left(N^{0}\right)-n f\left(\varepsilon^{*}\right)\right.$, $\left.f\left(\varepsilon^{*}\right), \ldots, f\left(\varepsilon^{*}\right)\right) \in \mathbb{R}^{n+1}$.

(ii) If $\varepsilon^{*}>n\left[E^{*}(0)-E^{*}(n-1)\right]$, then the nucleolus $\eta(v)=\left(v\left(N^{0}\right)-n E^{*}(n-1)\right.$, $\left.E^{*}(n-1), \ldots, E^{*}(n-1)\right) \in \mathbb{R}^{n+1}$.

Proof: Since $\eta(v) \in I(v)$ and $\eta_{i}(v)=\eta_{j}(v)$ for all $i, j \in N$, the nucleolus $\eta(v)$ is of the form $\left(v\left(N^{0}\right)-n \beta, \beta, \ldots, \beta\right) \in \mathbb{R}^{n+1}$ for a certain $\beta \in \mathbb{R}$. Shortly, $\eta(v)$ $=x(\beta)$ where $\beta \in \mathbb{R}$.

(i) Suppose $\varepsilon^{*} \leq n\left[E^{*}(0)-E^{*}(n-1)\right]$. From Theorem 4.4 (i) - (ii), we deduce that the equivalence $x(\alpha) \in C_{\varepsilon^{*}}(v) \cap I(v) \Leftrightarrow \alpha=f\left(\varepsilon^{*}\right)$ holds for any $\alpha \in \mathbb{R}$. From this we conclude that $\eta(v)=x(\beta)$ where $\beta=f\left(\varepsilon^{*}\right)$.

(ii) Suppose $\varepsilon^{*}>n\left[E^{*}(0)-E^{*}(n-1)\right]$. By Theorem 4.4 (iii), the set $C_{\bar{\varepsilon}}(v)$ $\cap I(v)$ is nonempty and it contains the unique symmetrical imputation $x(\alpha)$ where $\alpha=E^{*}(n-1)$. From this we conclude that $\eta(v)=x(\beta)$ where $\beta=$ $E^{*}(n-1)$. 
The nucleolus payoff $f\left(\varepsilon^{*}\right)$ to a producer reflects the situation in which the producer's maximal reward for his cooperative behaviour (with respect to the formation of coalitions consisting of producers only) matches exactly his minimal reward for his noncooperative behaviour (with respect to the formation of coalitions containing the initial owner of the information about the technological innovation). The individual rationality principle must always be met by the nucleolus and therefore, the nucleolus payoff to a producer equals his single worth $E^{*}(n-1)$ in case the amount $f\left(\varepsilon^{*}\right)$ is less than his single worth.

The remainder of the section deals with the "core" solution concept which was introduced in game theory in Gillies (1953). The core of any cooperative game consists of imputations that give rise only to nonpositive excesses and as such, the core can be regarded as the strong 0-core. As a matter of fact, the notion of a strong $\varepsilon$-core was presented as a generalization of the core (cf. Shapley and Shubik, 1963, 1966) because the core itself may be empty. It follows immediately from Theorem 3.2 that the nucleolus of any cooperative game is always included in the nonempty core. According to the next theorem, the nonemptiness of the core $C(v)$ for the information trading game $\left(N^{0} ; v\right)$ depends on the sign of the corresponding critical number $\varepsilon^{*}$ satisfying $f\left(\varepsilon^{*}\right)=$ $g\left(\varepsilon^{*}\right)$.

Theorem 5.2: Let $\left(N^{0} ; v\right)$ be the information trading game. Then $C(v) \neq \phi$ iff $\varepsilon^{*} \leq 0$.

Proof: (i) Suppose $\varepsilon^{*} \leq 0$ or equivalently, $f(0) \leq g(0)$. Lemma 4.2 yields that $x(\alpha) \in C(v)$ for all $\alpha \in \mathbb{R}$ with $f(0) \leq \alpha \leq g(0)$. Thus, $C(v) \neq \phi$ whenever $\varepsilon^{*} \leq 0$.

(ii) To prove the converse statement, suppose $C(v) \neq \phi$. Then we have $\eta(v) \in C(v)$, while the nucleolus $\eta(v)$ is of the form $x(\beta)$ where $\beta \in \mathbb{R}$. By applying Lemma 4.2 to $x(\beta) \in C(v)$, we obtain $f(0) \leq \beta \leq g(0)$. So, $C(v) \neq \phi$ implies $f(0) \leq g(0)$ or equivalently, $\varepsilon^{*} \leq 0$.

The core of the information trading game is nonempty if and only if the critical number $\varepsilon^{*}$ induces a bonus (the size of $-\varepsilon^{*}$ ) on the formation of any coalition different from the grand coalition. In other words, the information trading game possesses a nonempty core iff the potential nucleolus payoff $f\left(\varepsilon^{*}\right)$ to any producer exceeds his monetary profit level $E^{*}(0)$ obtained before the technological innovation.

Regarding the formation of the grand coalition $N^{0}$, we pay attention to the number of producers who purchase the new technology. Here we suppose that the information about the technological innovation is shared between the smallest number of producers in case there are more numbers for which the maximum in the expression of the worth $v\left(N^{0}\right)$ is attained. To be exact, we define the number of producers who actually acquire the new technology by 


$$
m:=\min \left(r \mid r \in\{0,1, \ldots, n\}, \quad r E(r)+(n-r) E^{*}(r)=v\left(N^{0}\right)\right)
$$

Next we mention two relations between the number $m$ and the nonemptiness of the core for the information trading game.

Theorem 5.3: Let $\left(N^{0} ; v\right)$ be the information trading game.

(i) If $C(v) \neq \phi$, then $m \neq 0$.

(ii) If $C(v) \neq \phi$ and $E^{*}(0)>E^{*}(1)$, then $m=n$.

Proof: (i) Suppose $C(v) \neq \phi$. By Theorem 5.2, we have $\varepsilon^{*} \leq 0$ or equivalently, $g(0) \geq f(0)$. It follows that $(n-s)^{-1}\left[v\left(N^{0}\right)-v\left(S^{0}\right)\right] \geq E^{*}(0)$ for all $S^{0} \varsubsetneqq N^{0}$. From $S^{0}=\{0,1\}$ we deduce $v\left(N^{0}\right) \geq E(1)+(n-1) E^{*}(0)$, whereas $E(1)>E^{*}(0)$ by Assumption A.4. We obtain the strict inequality $v\left(N^{0}\right)>n E^{*}(0)$ and therefore, $m \neq 0$.

(ii) Suppose $C(v) \neq \phi$ and $E^{*}(0)>E^{*}(1)$. We establish the equality $m=n$. Assume on the contrary $m \neq n$. Choose $x \in C(v)$ and let $i \in N$. From $m \in$ $\{0,1, \ldots, n-1\}$ we derive that $v\left(N^{0}-\{i\}\right) \geq m E(m)+(n-1-m) E^{*}(m)$, whereas $v\left(N^{0}\right)=m E(m)+(n-m) E^{*}(m)$ by definition of the number $m$. Together with $x \in C(v)$, this yields $x_{i}=v\left(N^{0}\right)-x\left(N^{0}-\{i\}\right) \leq v\left(N^{0}\right)-v\left(N^{0}-\{i\}\right) \leq E^{*}(m)$. So, $x_{i} \leq E^{*}(m)$ for all $i \in N$. Summing up over all $i \in N$, we obtain $x(N) \leq n E^{*}(m)$. Furthermore, $x(N) \geq v(N)=n E^{*}(0)$ because of $x \in C(v)$. It follows that $n E^{*}(m) \geq x(N) \geq n E^{*}(0)$ and therefore, $E^{*}(m) \geq E^{*}(0)>E^{*}(1)$. From this and Assumption A.3, we conclude that $m=0$. However, part (i) states that $m=0$ is in contradiction with $C(v) \neq \phi$.

Let the information trading game possess a nonempty core. Then Theorem 5.3 (i) expresses that at least one producer obtains the information about the technological innovation. Besides, the new technology is actually acquired by every producer whenever the profit structure satisfies the strict inequality $E^{*}(0)>E^{*}(1)$. Here the additional requirement simply says that an actual buyer of the new technology causes a strict decrease of the profit level of the nonbuyers.

\section{The Kernel of the Information Trading Game}

Analogous to the nucleolus, the kernel of any cooperative game possesses the property that symmetrical players in the game receive the same payoff according to a kernel element (cf. Maschler and Peleg, 1966). In particular, the payoffs by 
kernel elements to the symmetrical producers in the information trading game $\left(N^{0} ; v\right)$ satisfy $x_{i}=x_{j}$ for all $i, j \in N$ and all $x \in K(v)$. For that reason, we apply both Definition 3.3 and Theorem 3.4 to symmetrical strong $\varepsilon$-core elements in the information trading game. The obtained result states that the kernel $K(v)$ of the information trading game coincides with the nucleolus.

Lemma 6.1: Let $\varepsilon \in \mathbb{R}, \alpha \in \mathbb{R}$ and $x(\alpha) \in C_{\varepsilon}(v)$. Then

(i) $\delta_{i 0}(\varepsilon, x(\alpha))=\varepsilon-f^{-1}(\alpha)$ for all $i \in N$,

(ii) $\delta_{0 j}(\varepsilon, x(\alpha))=\varepsilon-g^{-1}(\alpha)$ for all $j \in N$.

Proof: By Lemma 4.2, we have $f(\varepsilon) \leq \alpha \leq g(\varepsilon)$. Let $i, j \in N^{0}$ be such that $i \neq j$. For any $\delta \in \mathbb{R}$, we define the vector $y^{\delta} \in \mathbb{R}^{n+1}$ by $y^{\delta}:=x(\alpha)-\delta e^{i}+\delta e^{j}$. Note that the inverse $f^{-1}\left(g^{-1}\right.$ respectively) of the function $f(g)$ exists because $f(g)$ is a strictly decreasing (increasing) function.

(a) Consider the case $i=0$. Suppose $\delta>\varepsilon-g^{-1}(\alpha)$ or equivalently, $g(\varepsilon-\delta)<\alpha$. Then there exists $S^{0} \varsubsetneqq N^{0}$ such that $(n-s)^{-1}\left[v\left(N^{0}\right)-v\left(S^{0}\right)+\varepsilon-\delta\right]<\alpha$. Without loss of generality, we may assume $j \notin S^{0}$ (otherwise, replace $S^{0}$ by the coalition $\left(S^{0} \cup\{k\}\right)-\{j\}$ of equal worth where $\left.k \in N^{0}-S^{0}\right)$. Now we obtain $y^{\delta}\left(S^{0}\right)=$ $v\left(N^{0}\right)+(s-n) \alpha-\delta<v\left(S^{0}\right)-\varepsilon$. From this we conclude that $y^{\delta} \notin C_{\varepsilon}(v)$ whenever $\delta>\varepsilon-g^{-1}(\alpha)$.

(b) Consider the case $i=0$. Suppose $\delta=\varepsilon-g^{-1}(\alpha)$ or equivalently, $g(\varepsilon-\delta)$ $=\alpha$. This yields $\alpha \leq(n-s)^{-1}\left[v\left(N^{0}\right)-v\left(S^{0}\right)+\varepsilon-\delta\right]$ for all $S^{0} \varsubsetneqq N^{0}$. It follows that $y^{\delta}\left(S^{0}\right)=v\left(N^{0}\right)+(s-n) \alpha-\delta \geq v\left(S^{0}\right)-\varepsilon$ for all $S^{0} \varsubsetneqq N^{0}$ satisfying $j \notin S^{0}$. From both $\delta \geq 0$ and $x(\alpha) \in C_{\varepsilon}(v)$, we deduce that $y^{\delta}(T) \geq v(T)-\varepsilon$ for all other $T \subset N^{0}, T \neq N^{0}, \phi$. We conclude that $y^{\delta} \in C_{\varepsilon}(v)$ whenever $\delta=\varepsilon-g^{-1}(\alpha)$. Together with part (a), this proves the statement (ii). It is left to the reader to establish the statement (i) by using similar reasonings as in the parts (a) - (b).

Theorem 6.2: The kernel $K(v)$ of the information trading game $\left(N^{0} ; v\right)$ is a singleton consisting of the nucleolus $\eta(v)$. That is, $K(v)=\{\eta(v)\}$.

Proof: Let $x \in K(v)$. Since $x \in I(v)$ and $x_{i}=x_{j}$ for all $i, j \in N$, the kernel element $x$ is of the form $\left(v\left(N^{0}\right)-n \beta, \beta, \ldots, \beta\right) \in \mathbb{R}^{n+1}$ for a certain $\beta \in \mathbb{R}$. Shortly, $x=x(\beta)$ where $\beta \in \mathbb{R}$. Choose the real number $\varepsilon$ large enough such that $x(\beta)$ $\in C_{\varepsilon}(v)$. By applying Theorem 3.4 to the vector $x(\beta)$, only one of the following three cases (1) - (3) must hold.

(1) $\delta_{01}(\varepsilon, x(\beta))=\delta_{10}(\varepsilon, x(\beta))$. In view of Lemma 6.1 , the relevant equality is equivalent to $f^{-1}(\beta)=g^{-1}(\beta)$, i.e., $\beta=f\left(\varepsilon^{*}\right)$. 
(2) $\delta_{01}(\varepsilon, x(\beta))>\delta_{10}(\varepsilon, x(\beta))$ and $x_{0}(\beta)=v(\{0\})$. This is equivalent to $f\left(\varepsilon^{*}\right)>\beta$ and $\beta=n^{-1} v\left(N^{0}\right)$. As such, the strict inequality $f\left(\varepsilon^{*}\right)>n^{-1} v\left(N^{0}\right)$ holds.

(3) $\delta_{01}(\varepsilon, x(\beta))<\delta_{10}(\varepsilon, x(\beta))$ and $x_{1}(\beta)=v((1))$. This is equivalent to $f\left(\varepsilon^{*}\right)<\beta$ and $\beta=E^{*}(n-1)$. As such, the strict inequality $f\left(\varepsilon^{*}\right)<E^{*}(n-1)$ holds.

Now we distinguish two cases as indicated in Theorem 5.1.

(i) Suppose $\varepsilon^{*} \leq n\left[E^{*}(0)-E^{*}(n-1)\right]$. It follows immediately from Theorem 4.4 (i) - (ii) that $x(\alpha) \in I(v)$ for $\alpha=f\left(\varepsilon^{*}\right)$. This yields $E^{*}(n-1) \leq f\left(\varepsilon^{*}\right)$ $\leq n^{-1} v\left(N^{0}\right)$. Regarding the above cases (1)-(3), we conclude that case (1) must hold. Hence, $\beta=f\left(\varepsilon^{*}\right)$ whenever $x(\beta) \in K(v)$.

(ii) Suppose $\varepsilon^{*}>n\left[E^{*}(0)-E^{*}(n-1)\right]$. Then $\varepsilon^{*}>0$ and so, $f\left(\varepsilon^{*}\right)=E^{*}(0)-$ $n^{-1} \varepsilon^{*}<E^{*}(n-1)$. Together with $x(\beta) \in I(v)$, this implies $\beta \geq E^{*}(n-1)>$ $f\left(\varepsilon^{*}\right)$. From $\beta>f\left(\varepsilon^{*}\right)$ we conclude that case (3) must hold. Hence, $\beta=$ $E^{*}(n-1)$ whenever $x(\beta) \in K(v)$.

Combining the results obtained in the cases (i) - (ii) and Theorem 5.1, we have established the inclusion $K(v) \subset\{\eta(v)\}$. Generally speaking, the kernel of any cooperative game always contains the nucleolus (cf. Schmeidler, 1969). Therefore, the equality $K(v)=\{\eta(v)\}$ is valid for the information trading game.

\section{The Information Trading Game with a Monopolistic Profit Structure}

This section is devoted to the study of the information trading game arising from a particular type of a profit structure. Firstly, the joint profit of any number of actual buyers of the new technology is supposed to be optimal in the situation with a unique buyer. Secondly, there are no profits for the non-buyers whenever a trade of the information has been carried out. Formally, the profit structure $\left\{E(t), E^{*}(t) \mid t \in\{0,1, \ldots, n\}\right\}$ of the information trading situation is said to be monopolistic if it satisfies the above two assumptions, i.e.,

(A.5) $E(1) \geq t E(t)$ for all $t \in\{1,2, \ldots, n\}$,

(A.6) $E^{*}(t)=0 \quad$ for all $t \in\{1,2, \ldots, n\}$.

The information trading game $\left(N^{0} ; v\right)$ with a monopolistic profit structure is as follows (cf. Definition 2.1):

$$
v\left(S^{0}\right):=\max \left(E(1), s E^{*}(0)\right) \text { for all } S^{0} \subset N^{0}, S^{0} \neq\{0\},
$$




$$
\begin{array}{ll}
v(S):=0 \quad \text { for all } S \subset N, S \neq N, & \\
v(\{0\}):=0, \quad v(N):=n E^{*}(0) .
\end{array}
$$

Notice that the relevant game $\left(N^{0} ; v\right)$ is zero-normalized (i.e., $v(\{i\})=0$ for all $i \in N^{0}$ ) and monotonic (i.e., $T_{1} \subset T_{2} \subset N^{0}$ implies $v\left(T_{1}\right) \leq v\left(T_{2}\right)$ ). The main results for the information trading situation with a monopolistic profit structure are derived from the exact determination of the critical number $\varepsilon^{*}$ satisfying $f\left(\varepsilon^{*}\right)=g\left(\varepsilon^{*}\right)$.

Proposition 7.1: Let $\left(N^{0} ; v\right)$ be the information trading game with a monopolistic profit structure.

(i) If $E(1) \leq n E^{*}(0)$, then $m=0, \varepsilon^{*}=(2 n-1)^{-1} n\left[E(1)-E^{*}(0)\right]$ and $f\left(\varepsilon^{*}\right)=$ $(2 n-1)^{-1}\left[2 n E^{*}(0)-E(1)\right]$.

(ii) If $E(1)>n E^{*}(0)$, then $m=1, \varepsilon^{*}=(2 n-1)^{-1} n(n-1) E^{*}(0)$ and $f\left(\varepsilon^{*}\right)=$ $(2 n-1)^{-1} n E^{*}(0)$.

Proof: (i) Suppose $E(1) \leq n E^{*}(0)$. Then we have $v\left(N^{0}\right)=n E^{*}(0)$ and so, $m=0$ by using the definition of the number $m$. Straightforward calculations yield that for all $\varepsilon \geq 0$

$$
g(\varepsilon)=\min \left(E^{*}(0)+n^{-1} \varepsilon,(n-1)^{-1}\left[n E^{*}(0)-E(1)+\varepsilon\right]\right)
$$

Put $\delta:=(2 n-1)^{-1} n\left[E(1)-E^{*}(0)\right]$. In view of Assumption A.4, the real number $\delta$ satisfies $0<\delta<n\left[E(1)-E^{*}(0)\right]$. From this and Lemma 4.3 (ii), it follows that $g(\delta)=(n-1)^{-1}\left[n E^{*}(0)-E(1)+\delta\right]$ and $f(\delta)=E^{*}(0)-n^{-1} \delta$. From $f(\delta)$ $=(2 n-1)^{-1}\left[2 n E^{*}(0)-E(1)\right]=g(\delta)$, we conclude that $\varepsilon^{*}=\delta$. This proves the statement (i).

(ii) Suppose $E(1)>n E^{*}(0)$. Then we have that $v\left(N^{0}\right)=v\left(S^{0}\right)=E(1)$ for all $S^{0} \subset N^{0}, S^{0} \neq\{0\}$. Straightforward calculations yield that for all $\varepsilon \geq 0$

$$
g(\varepsilon)=\min \left(n^{-1}[E(1)+\varepsilon],(n-1)^{-1} \varepsilon\right)
$$

Put $\gamma:=(2 n-1)^{-1} n(n-1) E^{*}(0)$. Now the real number $\gamma$ satisfies $0<\gamma<$ $(n-1) E(1)$ because $\gamma \leq n E^{*}(0)<E(1) \leq(n-1) E(1)$. It follows that $g(\gamma)=$ $(n-1)^{-1} \gamma$ and $f(\gamma)=E^{*}(0)-n^{-1} \gamma$. From $f(\gamma)=(2 n-1)^{-1} n E^{*}(0)=g(\gamma)$, we conclude that $\varepsilon^{*}=\gamma$. Furthermore, $v\left(N^{0}\right)=E(1)>n E^{*}(0)$ implies $m=1$. This proves the statement (ii). 
Theorem 7.2: Let $\left(N^{0} ; v\right)$ be the information trading game with a monopolistic profit structure. Then the core $C(v)=\phi$ and the nucleolus

$$
\eta(v)=\left(v\left(N^{0}\right)-n f\left(\varepsilon^{*}\right), \quad f\left(\varepsilon^{*}\right), \ldots, f\left(\varepsilon^{*}\right)\right) \in \mathbb{R}^{n+1}
$$

where

$$
\begin{aligned}
& f\left(\varepsilon^{*}\right)=(2 n-1)^{-1}\left[2 n E^{*}(0)-E(1)\right] \text { if } E(1) \leq n E^{*}(0) \text {, } \\
& =(2 n-1)^{-1} n E^{*}(0) \quad \text { if } E(1)>n E^{*}(0) .
\end{aligned}
$$

Proof: In view of the two Theorems 5.1 (i), 5.2 and Proposition 7.1, it suffices to establish the inequalities $0<\varepsilon^{*} \leq n\left[E^{*}(0)-E^{*}(n-1)\right]$. Because $E^{*}(n-1)=0$ for a monopolistic profit structure, the relevant inequalities reduce to $0<\varepsilon^{*}$ $\leq n E^{*}(0)$. We distinguish two cases.

(i) Suppose $E(1) \leq n E^{*}(0)$. By Proposition 7.1 (i), we get $\varepsilon^{*}=(2 n-1)^{-1} n$ $\times\left[E(1)-E^{*}(0)\right]$. From this and Assumption A.4, it is evident that $0<\varepsilon^{*}$ $\leq(2 n-1)^{-1} n(n-1) E^{*}(0) \leq n E^{*}(0)$.

(ii) Suppose $E(1)>n E^{*}(0)$. By Proposition 7.1 (ii), we get $\varepsilon^{*}=(2 n-1)^{-1} n$ $\times(n-1) E^{*}(0)$. Obviously, $0<\varepsilon^{*} \leq n E^{*}(0)$. In both cases, we conclude that the relevant inequalities $0<\varepsilon^{*} \leq n E^{*}(0)$ hold.

Proposition 7.1 (ii) expresses that, in case $E(1)>n E^{*}(0)$, the new technology is actually acquired by one of the producers. Under these circumstances, the monopolistic joint profit is shared between the initial owner of the information about the technological innovation and all of the producers in such a way that the profit share to the seller (according to the nucleolus) is more than the profit share to any producer. In case $E(1) \leq n E^{*}(0)$, the information about the new technology cannot be sold and the positive profit share $\varepsilon^{*}$ to the initial owner of the information can be interpreted as a compensation for not selling the information at all. Notice that the relevant compensation exceeds the profit share to any producer if and only if $E(1) \leq n E^{*}(0) \leq 3^{-1}(n+1) E(1)$.

\section{Concluding Remarks on Related Literature}

From a cooperative game theoretic viewpoint, we have analyzed a certain trade of the information about a technological innovation between the initial owner of the information and $n$ identical producers. Noncooperative game theoretic 
analyses of related information trading problems have been carried out in $\mathrm{Ka}$ mien and Tauman $(1984,1986)$ and Kamien, Tauman and Zang (1988). For instance, the noncooperative approach in Kamien and Tauman (1984) disclosed that the profit of any producer generally declines relative to his monetary profit level obtained before the technological innovation. In our cooperative setting, the same conclusion depends on the (non)emptiness of the core of the cooperative game model.

Earlier work on the information trading problem can be traced back to Arrow (1962). If the technological innovation is "drastic" in the sense of Arrow, then the noncooperative game models of the information trading problem degenerate into a monopoly (cf. Kamien and Tauman, 1986; Muto, 1987).

The characteristic function of our cooperative game model as presented in Definition 2.1 is strongly based on the two assumptions that the initial owner of the information about a technological innovation is not a producer and that resales of the new technology are completely prohibited. In case the initial owner of the information can also produce the output, then the information trading situation can be modelled as a cooperative game whose type is an example of the so-called "big boss games" (cf. Muto et al., 1988). If we relax the assumption of perfect patent protection and allow resales of the new technology, considerations will be necessary on what kind of coalitional behaviour is appropriate. Regarding this topic, Nakayama (1986) proposed a bargaining set-type solution. Cooperative game theoretic approaches in this direction would merit further study.

\section{References}

Arrow KJ (1962) Economic welfare and the allocation of resources for invention. In: Nelson RR (ed) The rate and direction of inventive activity. Princeton University Press, Princeton, New Jersey Davis M, Maschler M (1965) The kernel of a cooperative game. Naval Res Logist Quart 12:223 - 259

Gillies DB (1953) Some theorems on $n$-person games. Ph D Thesis. Princeton University Press, Princeton, New Jersey

Kamien MI, Tauman Y (1984) The private value of a patent: a game theoretic analysis. J Econom Suppl 4:93-118

Kamien MI, Tauman Y (1986) Fees versus royalties and the private value of a patent. Quart J Econom 101:471-491

Kamien MI, Tauman Y, Zang I (1988) Optimal license fees for a new product. Math Social Sciences 16:77- 106

Kats A, Tauman Y (1985) Coalition production economies with divisible and indivisible inputs. J Math Econom 14:19-42

Maschler M, Peleg B (1966) A characterization, existence proof and dimension bounds for the kernel of a game. Pacific J Math 18:289-328

Maschler M, Peleg B, Shapley LS (1979) Geometric properties of the kernel, nucleolus, and related solution concepts. Math Oper Res 4:303-338

Muto S (1987) Possibility of relicensing and patent protection. European Econom Rev 31:927-945 
Muto S, Nakayama M, Potters J, Tijs SH (1988) On big boss games. Econom Studies Quart 39:303-321

Nakayama M (1986) Bargaining for an information good with externalities. Working Paper no 79. Faculty of Economics, Toyama University, Toyama, Japan

Schmeidler D (1969) The nucleolus of a characteristic function game. SIAM J Appl Math $17: 1163-1170$

Shapley LS, Shubik M (1963) The core of an economy with nonconvex preferences. RM-3518, The Rand Corporation, Santa Monica, CA

Shapley LS, Shubik M (1966) Quasi-cores in a monetary economy with nonconvex preferences. Econometrica 34:805-827

Received: April 1990

Revised version received: November 1990 